review - well, up to a point. She showed it to seven of her senior $\mathrm{PhD}$ students, and then invited them to discuss it with her over a day of brainstorming, literature search and pizza. She channelled the resulting comments and criticisms into the referee's report that she submitted, and which all seven students saw again before submission. No confidence was breached during the review process: the students agreed to strict confidentiality, and Maniscalco opted in to our referee-accreditation trial scheme, which means that her name, together with those of the participating students, appears in a statement at the end of the research paper. Two other reviewers offered their thoughts on the paper in the usual way.

It is not unusual for a lab head to delegate a paper's peer review to a junior colleague, or to collaborate with one - and, unfortunately, the colleague's contribution isn't always acknowledged. It is unusual, as far as we know, to make peer review a team exercise and to openly state that that was how it was done. It's not an experiment we expect will be repeated often. Still, contrary to popular belief, our editors are, in principle, happy for referees to involve others in the review process, as long as confidentiality is assured and the editors are kept in the loop. It's right for all those involved in peer review to be acknowledged. At its best, such collaboration can enrich the review process and help junior researchers develop the skills needed to become effective referees themselves.

In this case, the paper explores the tension between quantum physics and local realism. The latter brings together two principles: locality - according to which, observing a particle at one physical location cannot have immediate effects on the properties of a particle at a different location - and realism, which expresses how the observable features of particles exist even if we don't actively measure them. But in quantum mechanics, correlations between distant particles exist that are so strong they violate local realism. Put differently, in quantum theory it is possible to have two correlated particles far away from each other, to measure the first and, as a result, learn something about the second without having observed it directly.

So here's the conundrum: does quantum mechanics really violate local realism, or could it be the case that some unknown factors would complete the theory and explain these apparent violations? In the 1960s, the physicist John Bell offered a way to tackle the problem in the laboratory, by studying quantum correlations in the form of entanglement.

"The BIG Bell
Test closes
the 'freedom-
of-choice'
loophole."
In these experiments, sequences of spatially separated measurements on entangled particles lead to computing a quantity that can have values not possible in the context of local and realistic theories. Bell tests have confirmed the validity of quantum theory many times, but they include assumptions that leave wiggle room for non-quantum explanations as to why local realism is violated, and so physicists have been looking for ways to close these loopholes ever since.

In 2015, physicists showed that successful Bell tests could not be due to speed-of-light communication between the particles, or to inefficient detection processes during the measurements. But another, more subtle, loophole was still open. Bell tests also assume that experimenters have free choice over which measurements they make on each particle. And yet, hidden parameters could be influencing these choices to produce correlations that give the illusion of entanglement.

The BIG Bell Test closes this freedom-of-choice loophole. The various experimental groups had no say in which measurement settings to use. Instead, they performed their measurements according to the unpredictable streams of bits received from the 100,000 gamers.

The results show the presence of correlations strong enough to contradict local realism. Maybe that's how 30 November 2016 might be remembered: the day the people of the world came together to test quantum theory.

\section{Helping hands}

\section{Awareness of mental health must be matched with steps such as better training for supervisors.}

$\mathrm{I}$ t's Mental Health Awareness Month in the United States, and next week is Mental Health Awareness Week in the United Kingdom. And awareness is certainly on the rise. Already this year, reports, surveys and studies have highlighted psychological struggles experienced by the old, the young, schoolchildren, men, women, soldiers, immigrants and refugees, football players, dancers, actors, social-media users, musicians, and elite athletes immediately after the Olympic Games. As quoted often, one in four people have a mental-health condition. And rates of depression and anxiety reported by postgraduate students are unacceptably high. This week, Nature is working to improve awareness of how mental illness can affect researchers: in this issue's Careers (page 267) and Comment (page 160) sections, several scientists share their experiences with honesty and admirable courage.

Awareness on its own is obviously not enough. John Lennon wrote that life is what happens while you're busy making other plans. Well, a life with mental illness can feel a lot like something that happens while well-meaning people are busy raising awareness. So, how do we make sure that those affected actually feel heard, supported and better?

Nature is trying to play a small part. Last month, we received a staggering response from readers to a Careers item about the alarmingly high rates of mental-health concerns reported by postgraduates. We invited people to tell us their stories, which we collected through a confidential online form. Our editors hoped to find some examples of success that we could share. Yet, almost without exception, the outpouring of 300-plus stories we received were from people who wanted support but were getting little, if any. (Of course, those who have found support might be less likely to tell their tale.) We printed five respondents' stories, with their kind permission, last week (Nature 557, 129-131;2018).

Most who replied were postgraduate students and postdocs, but several established scientists also wrote in to point out that mentalhealth problems are not confined to the young. We want to thank all those who responded so openly: it was harrowing reading, and will help to drive our future coverage of these issues. As many people struggling with their mental health eventually realize, it rarely helps to keep quiet. Reach out to someone and you'll probably be surprised at how readily they acknowledge what you're going through. Perhaps they are, too.

One problem is that, according to a report last year by RAND Europe, "the evidence around the effectiveness of interventions to support the mental health of researchers specifically is thin. Few interventions are described in the literature and even fewer of those have been evaluated" (see go.nature.com/2juanaw).

Some efforts are already under way to help postgraduates, beyond simply raising awareness. In March, the Higher Education Funding Council for England announced it would put a total of $£ 1.5$ million (US\$2 million) towards improving mental health at 17 universities. Several schemes will endeavour to better train and equip PhD supervisors to mentor their students. This is much needed: a dysfunctional supervisor-student relationship was a common complaint from many who wrote to us.

Manipal Academy of Higher Education in India has established an independent, confidential student-support centre so that students can come directly to ask professional psychologists for immediate help. Meanwhile, the Francis Crick Institute in London has more than 20 registered mental-health first aiders, who are trained to recognize mentalhealth issues, provide initial help and guide people towards professional services where appropriate. These and other examples of good practice and sources of support are collated in a dedicated page on our website (see go.nature.com/2i9a6yx). We hope they inspire more. 